SNUTP 93-71

\title{
Factorization in graviton interactions
}

\author{
S. Y. Choi, J. S. Shim, and H. S. Song \\ Center for Theoretical Physics and Department of Physics, \\ Seoul National University, Seoul 151-742, Korea
}

\begin{abstract}
The study of factorization in the linearized gravity is extended to the graviton scattering processes with a massive scalar particle, with a massless vector boson and also with a graviton. Every transition amplitude is shown to be completely factorized and the physical implications of their common factors are discussed.
\end{abstract}

Pacs numbers : 04.60.+n, 12.25.+e

Typeset using REVTEX 
Gravitational gauge invariance and graviton transversality force transition amplitudes of four-body graviton interactions to be factorized. Yang-Mills theories [1] also exhibit a similar factorization property.

In our previous work, we have shown that the transition amplitudes of $g e \rightarrow \gamma e$ [2] and $g e \rightarrow g e$ [3] processes are completely factorized into a kinematical factor, a fermion-QED Compton scattering form, and another gauge invariant term. In particular, the transition amplitude of graviton scattering with a massive fermion reads

$$
\begin{aligned}
\mathcal{M}_{g f} & =-\frac{\kappa^{2}}{2} F\left[\left(\epsilon_{1} \cdot \epsilon_{2}\right)+\frac{\left(p_{1} \cdot \epsilon_{1}\right)\left(p_{2} \cdot \epsilon_{2}\right)}{\left(p_{1} \cdot k_{1}\right)}+\frac{\left(p_{2} \cdot \epsilon_{1}\right)\left(p_{1} \cdot \epsilon_{2}\right)}{\left(p_{1} \cdot k_{2}\right)}\right] \\
& \times \bar{u}\left(p_{2}\right)\left[\frac{\phi_{2}\left(\not \not_{1}+\not k_{1}+m\right) \not_{1}}{2\left(p_{1} \cdot k_{1}\right)}+\frac{\phi_{1}\left(\not \not_{1}+\not k_{2}+m\right) \phi_{2}}{2\left(p_{1} \cdot k_{2}\right)}\right] u\left(p_{1}\right),
\end{aligned}
$$

where all particles are assumed to be incoming, the $\epsilon_{1}^{\mu} \epsilon_{1}^{\nu}\left(\epsilon_{2}^{\mu} \epsilon_{2}^{\nu}\right)$ and $k_{1}^{\mu}\left(k_{2}^{\mu}\right)$ are the wave tensor and four-momentum of the initial(final) graviton, and $p_{1}\left(p_{2}\right)$ is the four-momentum of the initial(final) fermion, respectively. The coupling is denoted by $\kappa=\sqrt{32 \pi G_{N}}$ and the overall kinematical factor $F$ is given by

$$
F=\frac{\left(p_{1} \cdot k_{1}\right)\left(p_{1} \cdot k_{2}\right)}{\left(k_{1} \cdot k_{2}\right)}
$$

The first bracket term in Eq. (1) is none other than the photon-scalar scattering amplitude and the second one is the photon-fermion scattering amplitude.

In this paper, we extend the study of factorization in the linearized gravity to the graviton scattering processes with a massive scalar, a massless vector boson, and a graviton itself.

Let us now introduce a manifestly gauge invariant four-vector $\tilde{\epsilon}_{i}(i=1,2)$ defined as

$$
\tilde{\epsilon}_{i}=\epsilon_{i}-\frac{\left(p_{1} \cdot \epsilon_{i}\right)}{\left(p_{1} \cdot k_{i}\right)} k_{i}
$$

As a result, the expression of photon-scalar scattering amplitude is converted into a greatly simplified form:

$$
\epsilon_{1} \cdot \epsilon_{2}+\frac{\left(p_{1} \cdot \epsilon_{1}\right)\left(p_{2} \cdot \epsilon_{2}\right)}{\left(p_{1} \cdot k_{1}\right)}+\frac{\left(p_{2} \cdot \epsilon_{1}\right)\left(p_{1} \cdot \epsilon_{2}\right)}{\left(p_{1} \cdot k_{2}\right)}=\tilde{\epsilon_{1}} \cdot \tilde{\epsilon_{2}}
$$


Along with this simplification, gravitational gauge invariance and graviton transversality render the transition amplitude $\mathcal{M}_{g s}$ of graviton-scalar scattering and the transition amplitude $\mathcal{M}_{g v}$ of graviton scattering with a massless vector boson completely factorized and simple:

$$
\mathcal{M}_{g s}=\frac{\kappa^{2}}{2} F\left[\tilde{\epsilon_{1}} \cdot \tilde{\epsilon_{2}}\right]^{2},
$$

and

$$
\begin{aligned}
& \mathcal{M}_{g v}=-\frac{\kappa^{2}}{2} F\left[\tilde{\epsilon_{1}} \cdot \tilde{\epsilon_{2}}\right]\left[\left(\tilde{\epsilon_{1}} \cdot \tilde{\epsilon_{2}}\right)\left(\tilde{\varepsilon_{1}} \cdot \tilde{\varepsilon_{2}}\right)+\left(k_{1} \cdot k_{2}\right)\left\{\frac{\left(\tilde{\epsilon_{1}} \cdot \tilde{\varepsilon_{1}}\right)\left(\tilde{\epsilon_{2}} \cdot \tilde{\varepsilon_{2}}\right)}{p_{1} \cdot k_{1}}+\frac{\left(\tilde{\epsilon_{1}} \cdot \tilde{\varepsilon_{2}}\right)\left(\tilde{\epsilon_{2}} \cdot \tilde{\varepsilon_{1}}\right)}{p_{1} \cdot k_{2}}\right\}\right. \\
& +\frac{1}{p_{1} \cdot k_{2}}\left\{\left(\tilde{\epsilon_{2}} \cdot \tilde{\varepsilon_{1}}\right)\left(p_{2} \cdot \tilde{\epsilon_{1}}\right)\left(k_{2} \cdot \tilde{\varepsilon_{2}}\right)+\left(\tilde{\epsilon_{1}} \cdot \tilde{\varepsilon_{2}}\right)\left(p_{2} \cdot \tilde{\epsilon_{2}}\right)\left(k_{2} \cdot \tilde{\varepsilon_{1}}\right)\right. \\
& \left.\left.-\left(\tilde{\epsilon_{2}} \cdot \tilde{\varepsilon_{2}}\right)\left(p_{2} \cdot \tilde{\epsilon_{1}}\right)\left(k_{2} \cdot \tilde{\varepsilon_{1}}\right)\right\}+\frac{1}{p_{1} \cdot k_{1}}\left(\tilde{\epsilon_{1}} \cdot \tilde{\varepsilon_{1}}\right)\left(p_{2} \cdot \tilde{\epsilon_{2}}\right)\left(k_{2} \cdot \tilde{\varepsilon_{2}}\right)\right] \text {, }
\end{aligned}
$$

where $p_{1}\left(p_{2}\right)$ and $\varepsilon_{1}\left(\varepsilon_{2}\right)$ are the four-momentum and wave vector of the initial(final) massless vector boson, respectively, and $\tilde{\varepsilon_{i}}(i=1,2)$ is defined in a gauge invariant form as

$$
\tilde{\varepsilon_{i}}=\varepsilon_{i}-\frac{\left(k_{1} \cdot \varepsilon_{i}\right)}{\left(k_{1} \cdot p_{i}\right)} p_{i} .
$$

The second bracket term of Eq. (66) corresponds to the transition amplitude of photon scattering with a massless charged vector boson. Let us employ a symbol $\mathcal{M}_{\gamma v}$ for the amplitude. Likewise, the transition amplitude of graviton-graviton scattering [4] turns out to be factorized as

$$
\mathcal{M}_{g g}=\frac{\kappa^{2}}{2} F\left[\mathcal{M}_{\gamma v}\right]^{2} .
$$

Here $p_{1}\left(p_{2}\right)$ and $\varepsilon_{1}^{\mu} \varepsilon_{1}^{\nu}\left(\varepsilon_{2}^{\mu} \varepsilon_{2}^{\nu}\right)$ are the four-momentum and wave tensor of another initial(final) graviton, respectively. While the form of Eq. (四) is independent of the choice of $\tilde{\epsilon}_{i}$, the photon-vector boson scattering amplitude $\mathcal{M}_{\gamma v}$ can be changed if the $\tilde{\epsilon}_{i}$ and $\tilde{\varepsilon}_{i}$ are defined in a different way. Nevertheless, the transition amplitude $\mathcal{M}_{g g}$ satisfies Bose and crossing symmetries. The completely symmetric expression of $\mathcal{M}_{g g}$ can be found in Ref. 四.

Several comments are in order. 
(a) The $\tilde{\epsilon}_{i}$ and $\tilde{\varepsilon}_{i}$ are manifestly gauge invariant and so are all four-body transition amplitudes.

(b) The helicity amplitudes [5.6] for the graviton-scalar, graviton-photon, and gravitongraviton scattering processes can be immediately obtained from our corresponding factorized transition amplitudes.

(c) The expression $\left[\tilde{\epsilon_{1}} \cdot \tilde{\epsilon_{2}}\right]$ always appears as a common factor in the graviton scattering with matter fields such as a massive scalar, a massive fermion, and a massless vector boson, while the $\mathcal{M}_{\gamma v}$ is a common factor in the graviton scattering processes with a massless vector boson and a graviton itself.

To conclude, every four-body graviton scattering amplitude is completely factorized and the introduction of manifestly gauge invariant four-vectors $\tilde{\epsilon_{i}}$ and $\tilde{\varepsilon_{i}}(i=1,2)$ renders the amplitude very simple. These two simplifications justify why, with all the very complicated three-graviton and four-graviton vertices [6,7], the final form of transition amplitudes is so simple. The factorization property looks so generic that its more intensive and extensive investigation is expected to provide us with some crucial clues for the unification of gravity with other interactions.

\section{ACKNOWLEDGMENTS}

The work was supported in part by the Korea Science and Engineering Foundation through the SRC program and in part by the Korean Ministry of Education 


\section{REFERENCES}

[1] C. J. Goebel, F. Halzen, and J. P. Leveille, Phys. Rev. D23, 2682 (1981); Zhu Dongpei, Phys. Rev. D22, 2266 (1980).

[2] S. Y. Choi, Jungil Lee, J. S. Shim, and H. S. Song, Phys. Rev. D48, 769 (1993).

[3] S. Y. Choi, J. S. Shim, and H. S. Song, Phys. Rev. D48, (Sept. 15 issue) (1993).

[4] S. Sannan, Phys. Rev. D34, 1749 (1986).

[5] M. T. Grisaru, P. van Nieuwenhuizen, and C. C. Wu, Phys. Rev. D12, 397 (1975); M. T. Grisaru, H. Pendleton, P. van Nieuwenhuizen, Phys. Rev. D15, 996 (1977); H. T. Cho and K. L. Ng, Phys. Rev. D47, 1692 (1993).

[6] F. A. Berends and R. Gastmans, Nucl. Phys. B88, 99 (1975).

[7] B. S. DeWitt, Phys. Rev. 162, 1239 (1967). 